\title{
PEP-1-CAT protects hypoxia/reoxygenation- induced cardiomyocyte apoptosis through multiple sigaling pathways
}

Lei Zhang ${ }^{1,2+}$, Shuang Wei ${ }^{1,3 \dagger}$, Jun-Ming Tang ${ }^{1,2}$, Ling-Yun Guo ${ }^{1,2}$, Fei Zheng ${ }^{1,2}$, Jian-Ye Yang ${ }^{1,2}$, Xia Kong ${ }^{1,2}$, Yong-Zhang Huang ${ }^{1,2}$, Shi-You Chen ${ }^{4}$ and Jia-Ning Wang ${ }^{1,2,3^{*}}$

\begin{abstract}
Background: Catalase (CAT) breaks down $\mathrm{H}_{2} \mathrm{O}_{2}$ into $\mathrm{H}_{2} \mathrm{O}$ and $\mathrm{O}_{2}$ to protects cells from oxidative damage. However, its translational potential is limited because exogenous CAT cannot enter living cells automatically. This study is aimed to investigate if PEP-1-CAT fusion protein can effectively protect cardiomyocytes from oxidative stress due to hypoxia/reoxygenation (H/R)-induced injury.

Methods: H9c2 cardomyocytes were pretreated with catalase (CAT) or PEP-1-CAT fusion protein followed by culturing in a hypoxia and re-oxygenation condition. Cell apoptosis were measured by Annexin V and PI double staining and Flow cytometry. Intracellular superoxide anion level was determined, and mitochondrial membrane potential was measured. Expression of apoptosis-related proteins including Bcl-2, Bax, Caspase-3, PARP, p38 and phospho-p38 was analyzed by western blotting.

Results: PEP-1-CAT protected H9c2 from H/R-induced morphological alteration and reduced the release of lactate dehydrogenase (LDH) and malondialdehyde content. Superoxide anion production was also decreased. In addition, PEP1-CAT inhibited H9c2 apoptosis and blocked the expression of apoptosis stimulator Bax while increased the expression of $\mathrm{BCl}$-2, leading to an increased mitochondrial membrane potential. Mechanistically, PEP-1-CAT inhibited p38 MAPK while activating PI3K/Akt and Erk1/2 signaling pathways, resulting in blockade of Bcl2/Bax/mitochondrial apoptotic pathway.

Conclusion: Our study has revealed a novel mechanism by which PEP-1-CAT protects cardiomyocyte from H/R-induced injury. PEP-1-CAT blocks Bcl2/Bax/mitochondrial apoptotic pathway by inhibiting p38 MAPK while activating $\mathrm{PI}$ IK/Akt and Erk1/2 signaling pathways.
\end{abstract}

Keywords: Cell-penetrating peptide, PEP-1, Catalase, Cardiomyocyte, Apoptosis, MAPK

\section{Introduction}

Myocardial ischemia and reperfusion generate a large amount of reactive oxygen species (ROS) in cardiomyocytes subject to injury. ROS assaults intracellular organelles, cell membranes, and biological macromolecules including nucleic acid, protein, and lipid, resulting in oxidative stress and cell apoptosis [1,2]. Catalase (CAT) is one of essential enzymes metabolizing oxygen free radical via breakdown of $\mathrm{H}_{2} \mathrm{O}_{2}$ into $\mathrm{H}_{2} \mathrm{O}$ and $\mathrm{O}_{2}$, and thus protects

\footnotetext{
* Correspondence: rywjn@vip.163.com

${ }^{\dagger}$ Equal contributors

'Institute of Clinical Medicine, Renmin Hospital, Hubei University of Medicine, Shiyan, Hubei 442000, China

${ }^{2}$ Department of Physiology and Key Lab of Human Embryonic Stem Cell of Hubei Province, Hubei University of Medicine, Hubei 442000, China Full list of author information is available at the end of the article
}

cells from oxidative damage. However, exogenous CAT does not enter living cells automatically because of its poor permeability and cell membrane selectivity. Its translational value in protecting cells from oxidative stress damage, therefore, is very limited.

A great deal of efforts have been made to deliver fulllength proteins into mammalian cells. Morris Group has designed a new type of PEP-1 peptide carrier (KETWWETWWTEWSQPKKKRKV) that enables the entering of large proteins into living cells [3]. In fact, several laboratories have successfully delivered fulllength PEP-1 fusion proteins into cultured cells and nervous system by using this PEP-1 peptide carrier, including EGFP, $\beta$-Gal, antibodies, cyclophilin $\mathrm{A}$, and human copper chaperone for $\mathrm{Cu}, \mathrm{Zn}$-SOD1 and CAT [4-7]. Our

\section{Biomed Central}


previous studies have demonstrated that PEP-1-CAT fusion proteins can be transduced into myocardium and protect against myocardial injury induced by ischemiareperfusion in rats [8].

Cardiomyocyte apoptosis is an inevitable process during myocardial ischemia-reperfusion-induced injury [9]. We have previously reported an anti-apoptotic effect of PEP-1-CAT on H9c2 cardiomyocytes [10]. However, detailed mechanisms underlying the effect of PEP-1-CAT on $\mathrm{H} / \mathrm{R}$-induced $\mathrm{H} 9 \mathrm{c} 2$ remain unknown. In the present study, we used the hypoxia-reoxygenation $(\mathrm{H} / \mathrm{R})$-induced apoptosis model to investigate the mechanisms underlying the anti-apoptotic effect of PEP-1-CAT in H9c2 cells. $\mathrm{H} / \mathrm{R}$ is a classic in vitro model mimicking myocardial ischemia-reperfusion injury in vivo. We found that PEP-1-CAT protected H9c2 from H/R-induced injury through blocking p38 MAPK activity and activating PI3K/Akt and Erk1/2 activity, which resulted in a blockade of $\mathrm{Bax} / \mathrm{Bcl}-2 /$ mitochondria apoptotic pathway and thus a reduction of cardiomyocyte apoptosis.

\section{Materials and methods}

Generation of biologically active PEP-1-CAT fusion protein PEP-1-CAT fusion protein was isolated and purified as described by our laboratory previously [11]. Briefly, two prokaryotic expression plasmids for CAT and PEP-1CAT were constructed using TA-cloning method. Both recombinant proteins were tagged with six histidine residues (His-tag) at the amino terminus. The two proteins were expressed and purified separately as described [11].

\section{Cell culture}

H9c2 cells were cultured in Dulbecco's modified Eagle's medium(DMEM,Invitrogen) with $5 \mathrm{~g} / \mathrm{L}$ glucose supplemented with $15 \%(\mathrm{v} / \mathrm{v})$ fetal bovine serum (FBS, Hangzhou sijiqing Biological Engineering Materials Co., Ltd., China). Cells were routinely grown to subconfluency ( $>90 \%$ by visual estimate) in $75 \mathrm{~cm}^{2}$ flasks at $37^{\circ} \mathrm{C}$ in a humidified atmosphere with $5 \% \mathrm{CO}_{2}$ prior to passage and seeding for experiments. To observe the morphological alteration, H9c2 cells were grown on cover slips and observed using a microscope (Nikon, Japan). To examine the aberrant nuclei in apoptotic cells, H9c2 cells were stained with 4,6Diamidino-2-phenylinole (DAPI), and the nuclei were observed using a fluorescent microscope.

\section{Immunocytochemistry staining}

$\mathrm{H} 9 \mathrm{c} 2$ cells were grown to confluence in a 24-well plate and treated with purified PEP-1-CAT $(2 \mu \mathrm{M})$ or CAT $(2 \mu \mathrm{M}) .6 \mathrm{~h}$ later, cells were washed twice with $1 \times \mathrm{PBS}$ and fixed with $4 \%$ paraformaldehyde for $15 \mathrm{~min}$ at room temperature. Immunocytochemistry staining was performed by using rabbit anti-Hisprobe (diluted 1:200) (Santa Cruz Biotechnology, USA) and mouse anti-
Troponin T antibodies (diluted 1:200) (Santa Cruz Biotechnology, USA). Cells were then incubated with tetraethyl rhodamine isothiocyanate (TRITC)-conjugated rat anti-rabbit Ig G (diluted 1:250) and fluorescein isothiocyanate (FITC)-conjugated goat anti-mouse Ig G (diluted $1: 250)$ at $25^{\circ} \mathrm{C}$ for $1 \mathrm{~h}$. After washing for 3 times with PBS, cells were incubated with DAPI (Sigma, USA) for $10 \mathrm{~min}$. The immunostained cells were observed with a fluorescent microscope (Nikon, Japan).

\section{Hypoxia-reoxygenation of H9c2 Cells}

$\mathrm{H} 9 \mathrm{c} 2$ cells were pretreated with or without PEP-1-CAT $(2 \mu \mathrm{M})$ in low serum media (2\% FBS) for $6 \mathrm{~h}$ followed by culturing in a low-oxygen condition $\left(95 \% \mathrm{~N}_{2}+5 \% \mathrm{CO}_{2}\right)$ for $21 \mathrm{~h}$ in a humidified hypoxia chamber (Stem Cell Technology, USA). After hypoxia incubation, the medium were replaced, and the cells were exposed to normaloxygen condition (95\% air $+5 \% \mathrm{CO}_{2}$ ) for reoxygenation for $6 \mathrm{~h} \mathrm{[12].} \mathrm{Control} \mathrm{cells} \mathrm{were} \mathrm{cultured} \mathrm{in} \mathrm{normoxic} \mathrm{con-}$ ditions. The supernatant and cells were collected separately for further analysis.

\section{Measurement of lactate dehydrogenase (LDH) and malondialdehy (MDA) levels}

H9c2 cells were treated with PEP-1-CAT, harvested and lysed as previously described LDH release and MDA content were measured using commercial kits (JianCheng Bioengineering Institute, China).

\section{Superoxide anion production in $\mathrm{H} 9 \mathrm{c} 2$}

$\mathrm{H} 9 \mathrm{c} 2$ cells were grown to confluence in a 24-well plate followed by $\mathrm{H} / \mathrm{R}$ with CAT or PEP-1-CAT treatment. Cells were then split and cultured on cover slips and incubated with DHE (5 mM) (Beyotime Insitute of Brotechnology) at $37^{\circ} \mathrm{C}$ for $30 \mathrm{~min}$. The DHE staining detecting superoxide anion production was observed using a fluorescent microscope (Nikon, Japan) or quantified by Flow Cytomety.

\section{Annexin V and PI binding assay}

Annexin V and PI fluorescein staining kit (Bender MedSystems, Austria) were utilized to measure H9c2 cell apoptosis by following the manufacturer's instruction. Briefly, $1 \times 10^{6}$ cells were suspended in $200 \mu \mathrm{l} 1 \times$ binding buffer (10 mM HEPES pH 7.4, $140 \mathrm{mM} \mathrm{NaCl}$, $2.5 \mathrm{mM} \mathrm{CaCl}_{2}$ ). Cells were then incubated with Annexin $\mathrm{V}$ (1:20) for $3 \mathrm{~min}$ followed by incubation with propidium iodide (PI, $1 \mathrm{mg} / \mathrm{ml}$ ) for $15 \mathrm{~min}$. Apoptosis rate was evaluated by Flow Cytometry.

\section{Measurement of mitochondrial membrane potential}

Mitochondrial transmembrane potential was assessed using a sensitive fluorescent dye, a lipophilic cationic probe JC-1 (Invitrogen, USA). H9c2 cells were grown on 
cover slips and incubated with $5 \mathrm{mM} \mathrm{JC-1}$ dye (Molecular Probes) at $37^{\circ} \mathrm{C}$ for $15 \mathrm{~min}$. Cells were then washed with PBS for three times and analyzed immediately with a fluorescent microscope. Red emission indicates membrane potential-dependent JC-1 aggregates in mitochondria. Green fluorescence reflects the monomeric form of JC-1 appearing in cytoplasm after mitochondrial membrane depolarization.

\section{Quantitative reverse transcription polymerase chain reaction (qPCR)}

Total RNA from H9c2 cells was extracted using TRIZOL Reagent (Invitrogen). RNA concentration was determined by UV spectrophotometry. qRT-PCR was performed using Thunderbird SYBR Master Mix (TOYOBO, Japan). Primer sequences were: Bcl-2: 5'-CGA CTT TGC AGA GAT GTC CA-3' (forward), 5'-ATG CCG GTT CAG GTA CTC AG-3' (reverse); Bax: 5' - CTG CAG AGG ATG ATT GCT GA-3' (forward), 5' - GAT CAG CTC GGG CAC TTT AG-3' (reverse); $\beta$-actin: $5^{\prime}$-GTC CAC CGC AAA TGC TTC TA-3' (forward), 5'-TGC TGT CAC CTT CAC CGT TC-3' (reverse). qPCR was performed on a Real-time PCR Detection System (Slan, Hongshi) with the following cycles: $95^{\circ} \mathrm{C}$ for $1 \mathrm{~min}$, followed by $95^{\circ} \mathrm{C}$ for $15 \mathrm{~s}, 58^{\circ} \mathrm{C}$ for $15 \mathrm{~s}$, and $72^{\circ} \mathrm{C}$ for $45 \mathrm{~s}$ for 40 cycles. $\beta$-actin expression was used as an internal control.

\section{Western blot analysis}

Western blot was carried out to detect protein expression using following primary antibodies: rabbit anti-Bax (Santa Cruz Biotechnology), mouse anti-Bcl-2 (Santa Cruz Biotechnology), rabbit anti-Caspase-3 (Santa Cruz Biotechnology), rabbit anti-PARP-1 (Santa Cruz Biotechnology), rabbit anti-phospho-p38 MAPK (cell signaling technology), and rabbit anti-p38 MAPK (cell signaling technology). The protein expression levels were visualized using enhanced chemiluminescence method.

\section{Statistical analysis}

All data are expressed as means \pm SEM unless indicated otherwise. Differences among groups were determined by ANOVA. Differences between groups were determined by Student's $t$-test with $\mathrm{P}<0.05$ considered statistically significant.

\section{Results}

PEP-1-CAT restored H/R-altered H9c2 cell morphology and decreased LDH and MDA levels

PEP-1-CAT fusion proteins were successfully transduced into H9c2 cells as shown by immunocytochemistry staining (Additional file 1: Figure S1). CAT fusion proteins, however, were unable to be transduced into the cells (Additional file 1: Figure S1). H/R altered H9c2 cell morphology. H/R treatment changed the spindle-shaped and well-organized morphology to a shrink, round and distorted morphology. PEP-1-CAT transduction, however, almost restored the spindle-shaped morphology seen in the untreated cells (Figure 1A).

$\mathrm{LDH}$ release is an indicator of cellular injury. Compared to untreated cells, LDH levels were markedly increased by $H / R$ injury. CAT transduction decreased LDH release. PEP-1-CAT transduction, however, had a greater impact on LDH levels compared to the CAT transduction (Figure 1B). MDA reflects cardiomyocyte oxidative damage. $H / R$ treatment strikingly increased the MDA level, but PEP-1-CAT significantly decreased the MDA level (Figure 1C).

\section{PEP-1-CAT had a greater effect on superoxide anion production than CAT}

$H / R$ treatment significantly increased superoxide anion production in H9c2 cells compared to the untreated group. CAT transduction slightly reduced superoxide anion level. PEP-1-CAT transduction, however, significantly inhibited the level of superoxide anion. These results demonstrated that PEP-1-CAT had a much stronger effect than CAT on removing superoxide anion from the injured cells (Figure 2).

\section{PEP-1-CAT attenuated H/R-induced H9c2 cell apoptosis}

Comparing to the control group, significantly more cells underwent apoptosis as shown by the bright DAPI staining in $H / R$ group. $H / R$ treatment condensed the nuclei of H9c2 cells, an indicator of apoptosis. PEP-1-CAT transduction, however, restored $\mathrm{H} 9 \mathrm{c} 2$ nuclei to the normal morphology (Figure 3A). Quantitative analysis using Flow Cytometry confirmed that PEP-1-CAT significantly inhibited H/R-induced apoptosis (Figure 3B-C). PolyADPribose polymerase-1 (PARP-1) is known to be involved in DNA damage while caspase- 3 is known to regulate cell apoptosis. To determine whether PEP-1-CAT affects H/Rinduced PARP and caspase- 3 cleavage, we treated cells with $H / R$ in the presence and absence of PEP-1-CAT and analyzed their cleavages using anti-PARP-1 and Caspase-3 antibodies. As shown in Figure 3D, H/R induced PARP and caspase- 3 cleavage in $\mathrm{H} 9 \mathrm{c} 2$ cells but the effects were inhibited by PEP-1-CAT, further demonstrating that PEP1-CAT suppressed H/R-induced apoptosis.

\section{PEP-1-CAT regulated the expression of apoptosis-related proteins}

To investigate the mechanism whereby PEP-1-CAT attenuates $\mathrm{H} / \mathrm{R}$-induced $\mathrm{H} 9 \mathrm{c} 2$ apoptosis, we examined the expression of Bcl-2 and Bax. Both qRT-PCR and Western blot analyses showed that $\mathrm{Bcl}-2$ expression was markedly increased in PEP-1-CAT-pretreated cells compared to the $\mathrm{H} / \mathrm{R}$ or CAT-treated group. As expected, Bax 

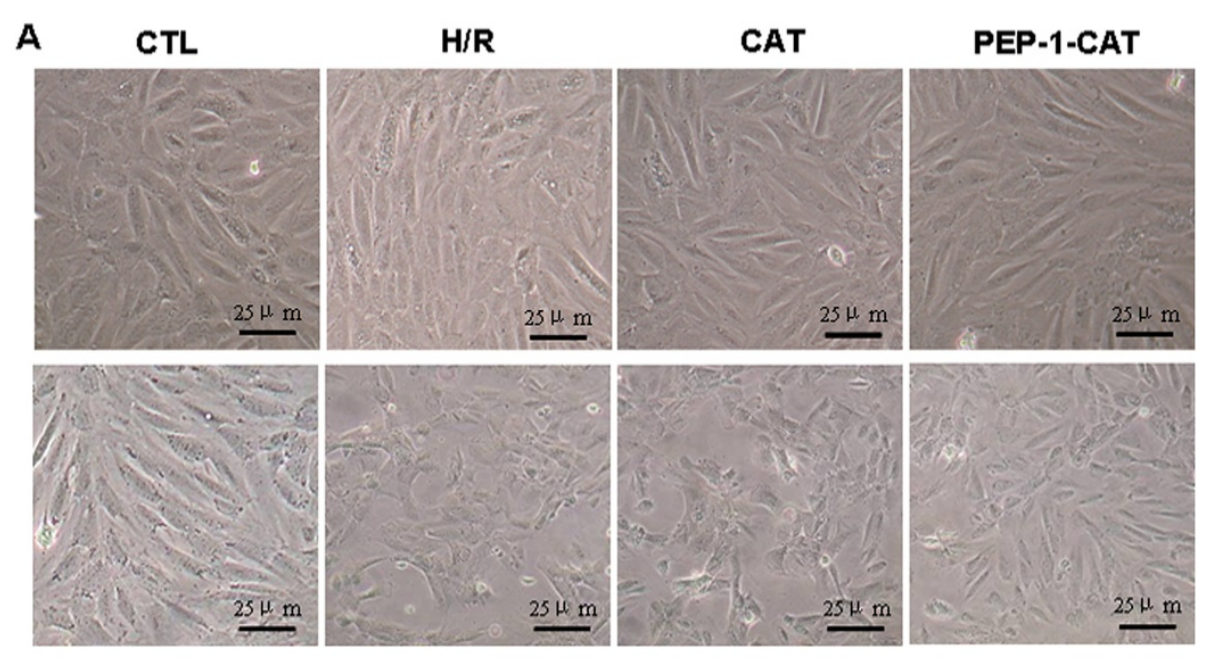

B

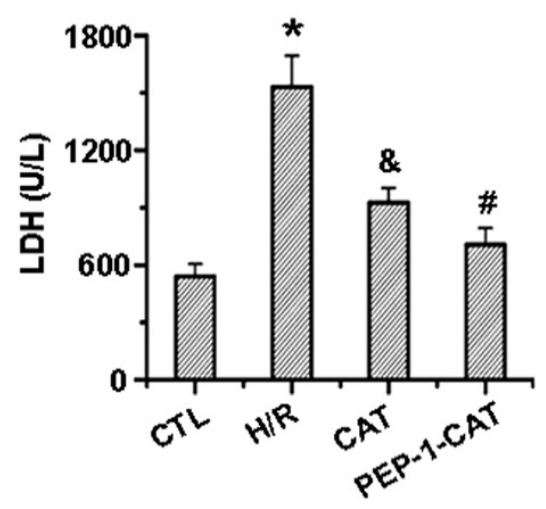

C

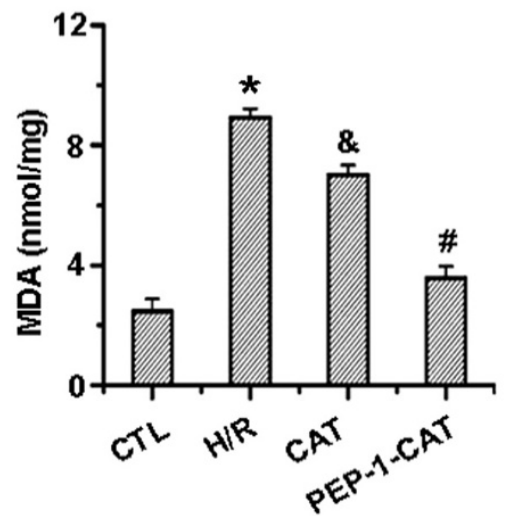

Figure 1 PEP-1-CAT restored H/R-induced alteration of $\mathrm{H} 9 \mathrm{C} 2$ cell morphology while inhibiting $\mathrm{H} / \mathrm{R}$-induced LDH release and MDA production. (A) Effect of PEP-1-CAT on H9c2 cell morphology. Upper panel: cell morphology prior to H/R treatment; lower panel: cell morphology with $H / R$ treatment. (B) PEP-1-CAT reduced LDH levels ${ }^{*} \mathrm{P}<0.01$ vs control $(C T L) ;{ }^{\circledR} \mathrm{P}<0.05 \mathrm{vs} H / \mathrm{R}$; ${ }^{\#} \mathrm{P}<0.01$ vs $H / \mathrm{R}$ or $H / R+C A T$ (CAT); $n=6$. (C) PEP-1-CAT decreased MDA levels. ${ }^{*} P<0.01$ vs control $(C T L){ }_{i}^{\&} P<0.05$ vs $H / R ;{ }^{*} P<0.01$ vs $H / R$ or $H / R+C A T(C A T) ; n=6$.

expression was markedly decreased by PEP-1-CAT (Figure 4A-D), suggesting that PEP-1-CAT prevented H9c2 cells from apoptosis by increasing Bcl-2 while inhibiting Bax expression.

\section{PEP-1-CAT restored H/R-blocked mitochondrial membrane potential}

Untreated cells exhibited bright-staining mitochondria that emitted red fluorescence. $H / R$ treatment caused the formation of monomeric JC-1, indicative of loss of membrane potential. PEP-1-CAT pretreatment, however, blocked the HR-induced formation of JC-1 monomers (Figure 5A-B), suggesting PEP-1-CAT can restore H/Rinduced loss of mitochondrial membrane potential.

PEP-1-CAT inhibited H/R-induced H9c2 apoptosis through regulating multiple signaling pathways

Previous studies have shown that apoptosis is mediated by multiple signaling pathways or protein factors including PI3K/Akt, p38 and Erk1/2 MAPK, etc. $[13,14]$. To determine which pathways are involved in PEP-1-CAT-mediated protection of H/R-injured H9c2 cells, we treated $\mathrm{H} 9 \mathrm{c} 2$ with specific inhibitors for each individual pathways. We found that PI3K/Akt and Erk1/2 signaling pathways were essential for mediating PEP-1-CAT inhibition of H/R-induced apoptosis because PI3K/Akt inhibitor wortmannin, PI3K siRNA, Erk1/2 inhibitor PD98059, or Erk1 siRNA blocked PEP-1CAT-induced reduction of H9c2 apoptosis (Figure 6A-B). p38 MAPK appeared to be also important for PEP1-CAT function. Although p38 MAPK inhibitor did not reverse PEP-1-CAT-mediated decrease of $\mathrm{H} 9 \mathrm{c} 2$ apoptosis (Figure 6A-B), PEP-1-CAT transduction inhibited p38 phosphorylation (Figure 7), suggesting that PEP-1-CAT blocks p38 signaling. These results demonstrated that PEP-1-CAT attenuated p38 signaling while enhancing PI3K and Erk1/2 MAPK signaling. 


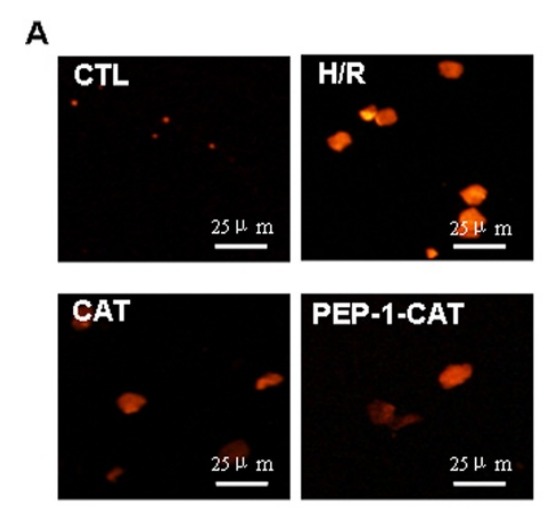

B

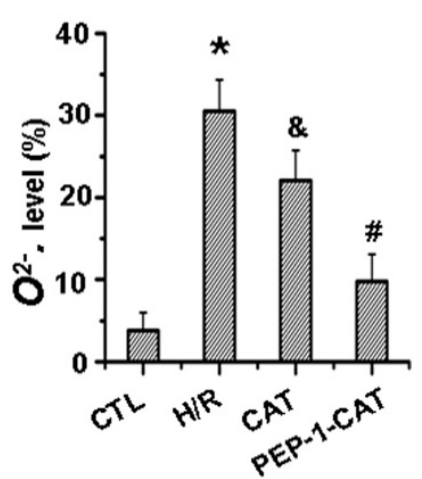

Figure 2 PEP-1-CAT inhibited H/R-induced superoxide anion production. (A) Superoxide anion production was observed using a fluorescent microscope. (B) Superoxide anion production was quantified by Flow Cytometry $(n=6)$. ${ }^{*} \mathrm{P}<0.01$ vs control $(C T L) ;{ }^{*} \mathrm{P}<0.05 \mathrm{vs} \mathrm{H/R}$; ${ }^{\#} \mathrm{P}<0.05$ vs $H / R$ or $\mathrm{H} / \mathrm{R}+\mathrm{CAT}(\mathrm{CAT}) ; \mathrm{n}=4$.

\section{Discussion}

Myocardial apoptosis is a significant pathophysiological event in myocardial ischemia-reperfusion injury [9]. It is widely acknowledged that intervention of myocardial apoptosis is a very important approach to the prevention of myocardial ischemia-reperfusion injury [15]. Reperfusion causes myocardium to produce a large amount of ROS including superoxide anion $\left(\mathrm{O}_{2}^{-} \cdot\right)$, hydroxyl radical
$\left(\mathrm{OH}^{-}\right)$, and hydrogen peroxide $\left(\mathrm{H}_{2} \mathrm{O}_{2}\right)$, etc [16]. CAT, one of most important enzymes, can protect cells from oxidative damage. But its potential to be used to protect myocardium from $\mathrm{H} / \mathrm{R}$-induced injury is hindered by the poor permeability and the selectivity of cell membrane. By fusing CAT with a PEP-1 peptide, we were able to efficiently transduce PEP-1-CAT into H9c2 cells and protect myocardium from $\mathrm{H} / \mathrm{R}$-induced injury [10]. The
A

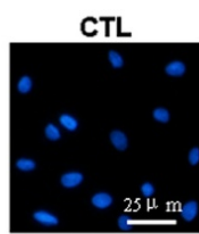

B

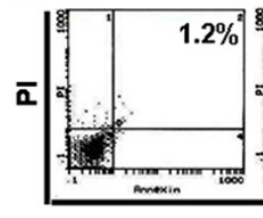

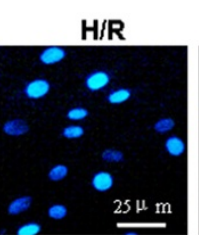
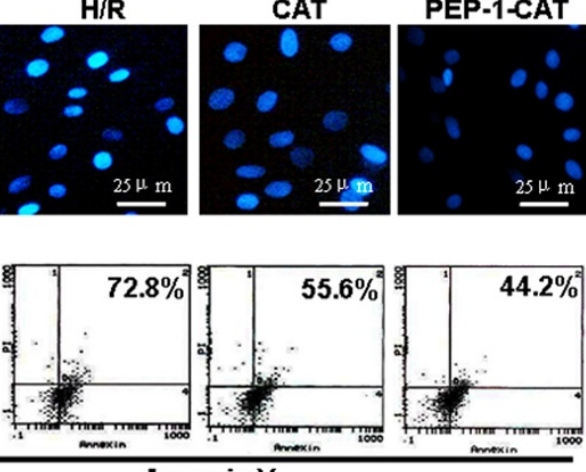

Annexin V

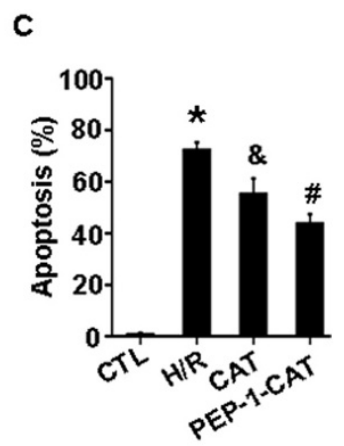

D

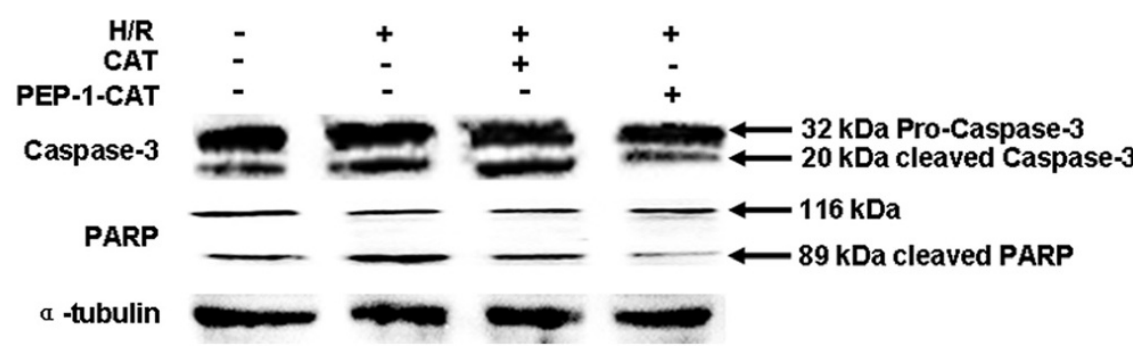

Figure 3 PEP-1-CAT inhibited H/R-induced H9c2 cell apoptosis. H9c2 cells were pretreated with CAT or PEP-1-CAT for 6 h and placed into normoxia environment for $27 \mathrm{~h}$ or into hypoxia chamber for $21 \mathrm{~h}$ followed by $6 \mathrm{~h}$ reoxygenation. (A) Cell apoptosis was detected by DAPI staining. (B-C) H/R-induced H9c2 apoptosis rate was quantiied by Flow Cytometry. ${ }^{*} \mathrm{P}<0.01$ vs control $(C T L) ;{ }^{\&} \mathrm{P}<0.05$ vs $H / R$; ${ }^{\#} \mathrm{P}<0.01$ vs $H / R$ or $H / R+C A T$ (CAT); $n=5$. (D) PARP and caspase-3 protein expression was detected by western blot. 


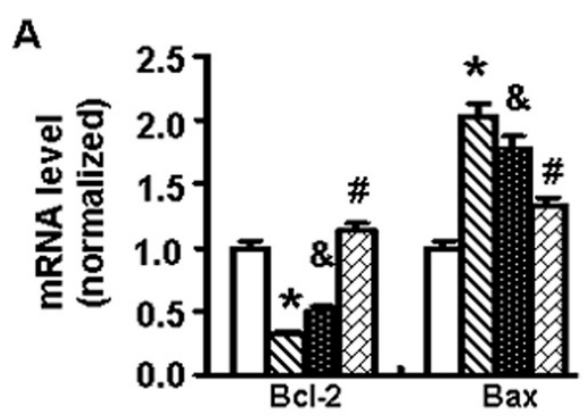

B

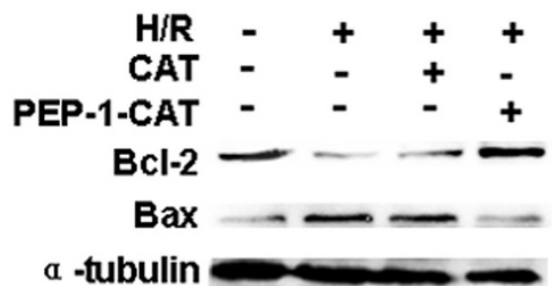

C
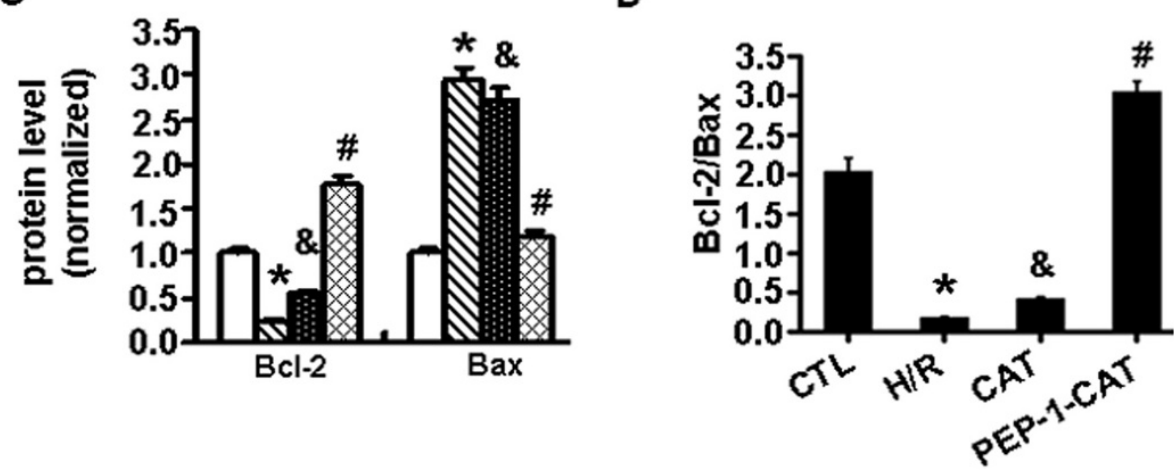

Figure 4 PEP-1-CAT regulated Bcl-2 and Bax expression. (A) Bcl2 and Bax mRNA expression was detected by qRT-PCR and normalized to $\beta$ actin. ${ }^{*} \mathrm{P}<0.01$ vs control (CTL); ${ }^{~} \mathrm{P}<0.05$ vs $H / R$; ${ }^{*} \mathrm{P}<0.05$ vs $\mathrm{H} / \mathrm{R}$ or $\mathrm{H} / \mathrm{R}+\mathrm{CAT}(\mathrm{CAT}) ; \mathrm{n}=3$. (B) $\mathrm{B} \mathrm{Cl} 2$ and Bax protein expression was detected by western blot. (C) Normalization of $B C l 2$ and Bax expression to a-Tubulin. ${ }^{*} P<0.01$ vs control $(C T L) ;{ }^{\circledR} P<0.05$ vs $H / R ;{ }^{*} P<0.05$ vs. $H / R$ or $H / R+C A T$

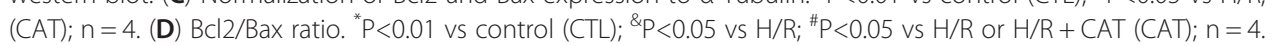

\section{A}
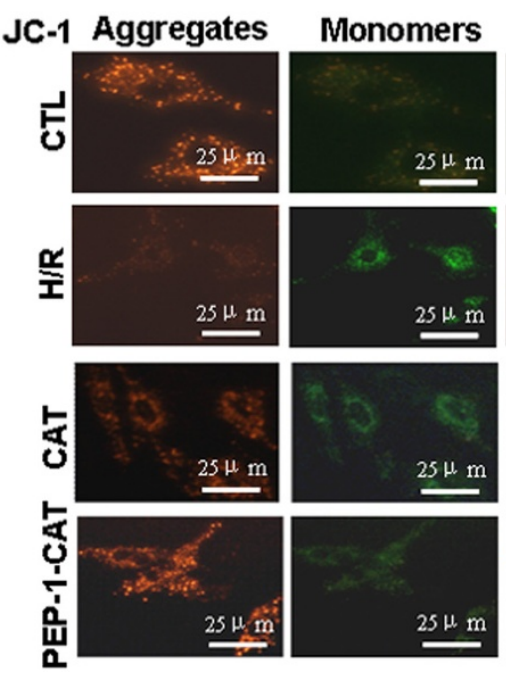

B
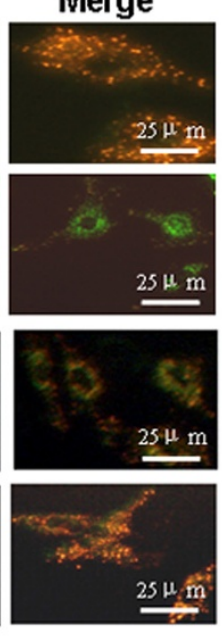

Figure 5 PEP-1-CAT restored H/R-induced reduction of mitochondrial membrane potential. (A) Mitochondrial transmembrane potential was assessed by the lipophilic cationic probe JC-1. Red signal indicates JC-1 aggregates in mitochondria. Green signal shows cytosolic JC-1 monomers indicative of the loss of mitochondrial membrane potential. (B) Quantitative analysis of membrane potential in (A). ${ }^{*} P<0.05$ vs control (CTL); ${ }^{\circledR} \mathrm{P}<0.05$ vs $H / R ;{ }^{*} \mathrm{P}<0.05$ vs $\mathrm{H} / \mathrm{R}$ or $\mathrm{H} / \mathrm{R}+\mathrm{CAT}$ (CAT) group; $\mathrm{n}=3$. 
A

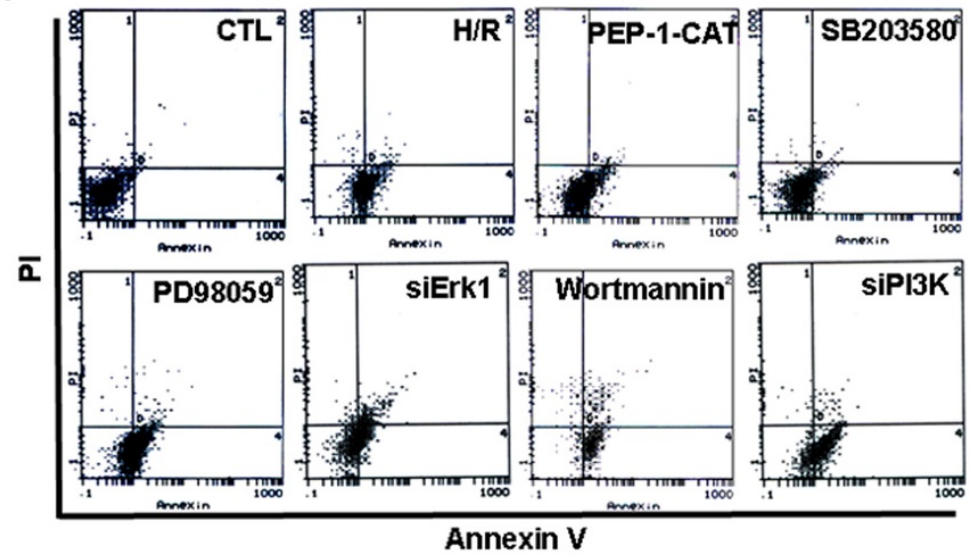

B

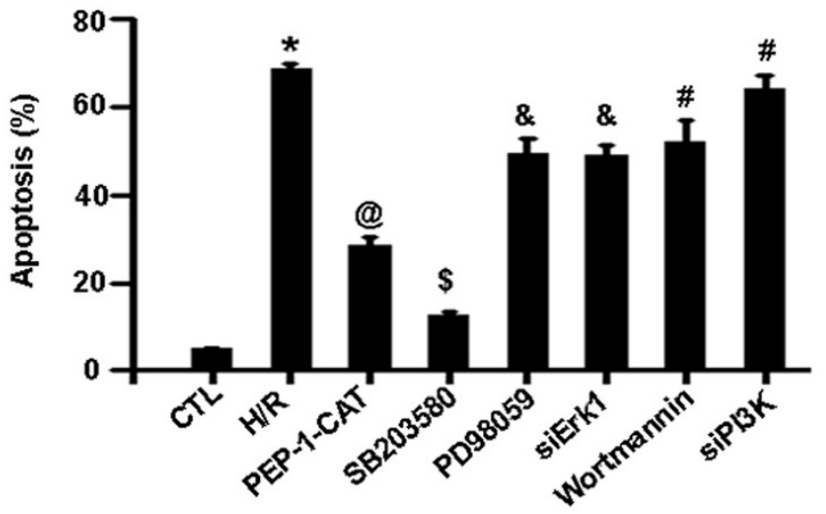

Figure 6 PEP-1-CAT inhibited H/R-induced H9c2 apoptosis via p38, PI3K and Erk1/2 MAPK signaling pathways. (A) H9c2 apoptosis was assessed by Flow Cytometry. (B) Quantification of H9c2 apoptosis rate. ${ }^{*} \mathrm{P}<0.01$ vs $C T L ;$; $\mathrm{P}<0.01 \mathrm{vs} \mathrm{H/R}$ group; ${ }^{\$} \mathrm{P}<0.01$ vs H/R or PEP-1-CATtreated group; ${ }^{\circledR} \mathrm{P}<0.05$ vs PEP-1-CAT-treated group; ${ }^{*} \mathrm{P}<0.05$ vs PEP-1-CAT-treated group; $n=5$.

present study advanced our previous finding by identifying novel mechanisms underlying PEP-1-CAT function in protecting cardiomyoctyes. We have found that PEP-1-CAT protects $\mathrm{H} / \mathrm{R}$-induced injury of $\mathrm{H} 9 \mathrm{c} 2$ cells by restoring $\mathrm{H} / \mathrm{R}$-induced alteration of $\mathrm{H} 9 \mathrm{c} 2$ morphology, inhibiting $\mathrm{H} / \mathrm{R}$-induced production of $\mathrm{O}_{2}^{-} \cdot$, and blocking $\mathrm{LDH}$ release and MDA production, the two indicators for hypoxia-reoxygenation injury $[17,18]$.

ROS causes damages to intracellular macromolecules such as DNA breakage and lipid membrane peroxidation, leading to cell apoptosis [19]. Our data demonstrate that PEP-1-CAT blocks H/R-induced H9c2 apoptosis by regulating mitochondria-related apoptotic pathways. Recent

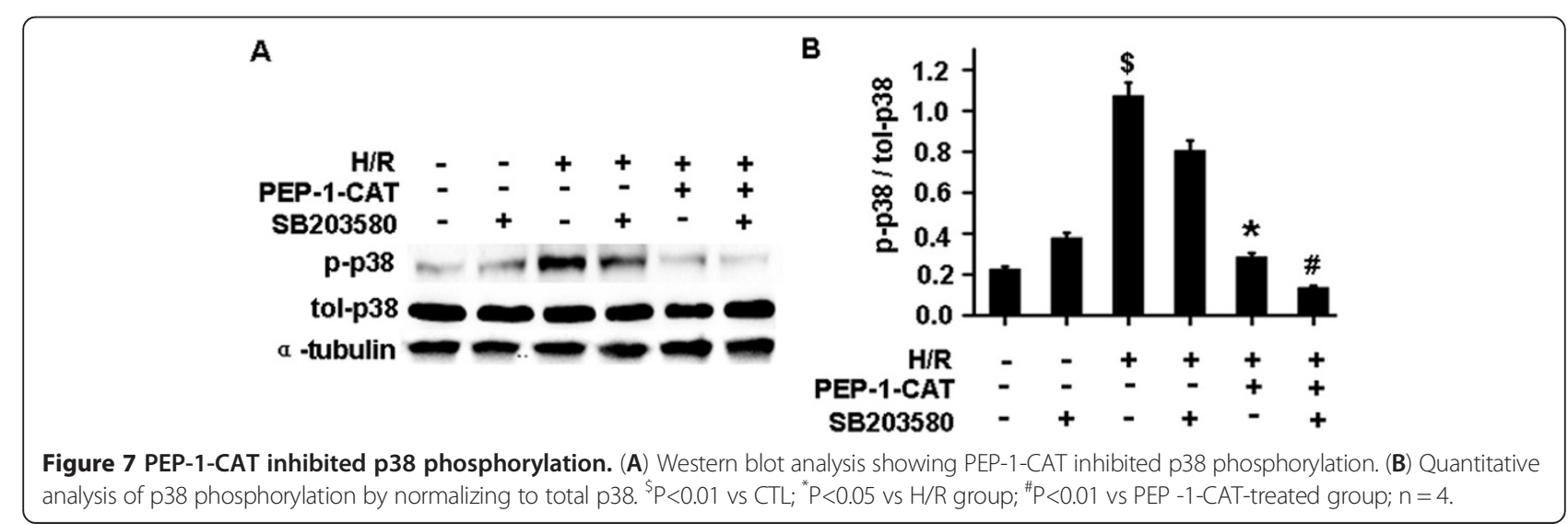


studies have shown that $H / R$ injury induces mitochondria to produce a high level of ROS [20,21]. Excessive ROS damages mitochondria, opens its permeability transition pore (PTP) and thus induces mitochondrial permeability transition (MPT), leading to mitochondrial depolarization and outer membrane rupture, which causes cell apoptosis or death $[22,23]$. Our studies indicate that $H / R$ induces a decreased mitochondrial membrane potential, suggesting an impairment of mitochondria function. PEP-1-CAT transduction, however, restores mitochondrial membrane potential. These data demonstrate that PEP-1-CAT protects H9c2 cells from H/R-induced apoptosis by maintaining mitochondria membrane integrity and function of cardiomyocytes. Moreover, previous studies indicate that $\mathrm{Bcl}-2$ family is upregulated during the opening of PTP [24]. Our results demonstrate that PEP-1-CAT regulates the expression of Bcl-2 family. PEP-1-CAT significantly increases $\mathrm{Bcl}-2$ while decreasing Bax protein levels that are altered by $\mathrm{H} / \mathrm{R}$ injury.

PEP-1-CAT prevents cardiomyocyte from H/R-induced injury by regulating multiple signaling pathways. Although a number of signaling pathways are involved in H/Rinduced myocardial injury and apoptosis, PEP-1-CAT protects cardiomyocytes through down-regulation of $\mathrm{p} 38$ MAPK and activation of PI3K and Erk1/2 signaling pathways. PEP-1-CAT transduction inhibits $\mathrm{p} 38$ MAPK phosphorylation, suggesting that $\mathrm{p} 38$ MAPK mediates, at least in part, the function of PEP-1-CAT. Blockade of PI3K and Erk1/2 signaling significantly attenuates PEP-1-CATmediated reduction of $\mathrm{H} 9 \mathrm{c} 2$ apoptosis, indicating that PI3K and Erk1/2 signaling pathways are essential for PEP1-CAT activity in protecting cardiomocytes.

In summary, PEP-1-CAT transduction efficiently protects cardiomyocyte from H/R-induced apoptosis by blocking ROS production in mitochondria, which maintains mitochondria membrane integrity and inhibits the activation of $\mathrm{Bcl} 2 / \mathrm{Bax}$ apoptotic pathway. Moreover, PEP1-CAT blocks cardiomyocyte apoptosis by blocking p38 MAPK while activating PI3K and Erk1/2 MAPK signaling pathways. How these signaling pathways interact with each other in mediating PEP-1-CAT function will be a interesting subject for future study. Nevertheless, our study provides novel information and rationale for developing PEP-1-CAT as a therapeutic agent for treating or preventing myocardial ischemia-reperfusion injury.

\section{Additional file}

Additional file 1: Figure S1. Transduction of PEP-1-CAT into H9c2 cells.

\section{Competing interests}

The authors declare that they have no competing interests.

\section{Authors' contributions}

LZ and SW designed and performed the experiments, collected the data and analyzed the results. JNW and JMT participated in the experimental design and interpretation of the results. LYG made fusion protein and evaluated the apoptosis by Flow Cytometry. FZ carried out Western blot. XK performed part of the in vitro experiments. JYY and $Y Z H$ carried out the immunoassays. SYC assisted with writing the manuscript. All the authors have read and approved the final manuscript.

\section{Authors' information}

Co-first author: Lei Zhang and Shuang Wei.

\section{Acknowledgements}

This study was supported by grants from National Natural Science Foundation of China (81170095), Hubei Education Department Science Foundation (T200811, T201112), China and National Institutes of Health (HL093429 and HL107526 to S.Y.C.).

\section{Author details}

${ }^{1}$ Institute of Clinical Medicine, Renmin Hospital, Hubei University of Medicine, Shiyan, Hubei 442000, China. ${ }^{2}$ Department of Physiology and Key Lab of Human Embryonic Stem Cell of Hubei Province, Hubei University of Medicine, Hubei 442000, China. ${ }^{3}$ Institute of Clinical Medicine and Department of Cardiology, Renmin Hospital, Hubei University of Medicine, Shiyan, Hubei 442000, China. ${ }^{4}$ Department of Physiology \& Pharmacology, University of Georgia, Athens, GA 30602, USA.

Received: 28 September 2012 Accepted: 1 May 2013

Published: 6 May 2013

\section{References}

1. Droge W: Free radicals in the physiological control of cell function. Physiol Rev 2002, 82:47-95.

2. Ahn HJ, Kim Kl, Kim G, Moon E, Yang SS, et al: Atmospheric-pressure plasma jet induces apoptosis involving mitochondria via generation of free radicals. PLoS One 2011, 6:e28154.

3. Morris MC, Depollier J, Mery J, Heitz F, Divita G: A peptide carrier for the delivery of biologically active proteins into mammalian cells. Nat Biotechnol 2001, 19:1173-1176.

4. Henriques ST, Costa J, Castanho MA: Translocation of beta-galactosidase mediated by the cell-penetrating peptide pep- 1 into lipid vesicles and human HeLa cells is driven by membrane electrostatic potential. Biochemistry 2005, 44:10189-10198.

5. Wu Y, Wood MD, Tao Y, Kataqiri F: Direct delivery of bacterial avirulence proteins into resistant Arabidopsis protoplasts leads to hypersensitive cell death. Plant J 2003, 33:131-137.

6. Zhang YE, Fu SZ, Li XQ, Chen P, Wang JL, et al: PEP-1-SOD1 protects brain from ischemic insult following asphgxial cardiac arrest in rats. Resuscitation 2011, 82:1081-1086.

7. Lee MJ, Kim DW, Sohn EJ, Jeong HJ, Shin MJ, et al: Anti-inflammatory effect of transduced PEP-1-cyclophilin A in Raw264.7 cells and 12-O-tetradecanoylphorbol-13-acetate-induced mice. Life Sci 2011, 89:896-904.

8. Zhang YJ, Wang JN, Tang JM, Huang YZ, Yang JY, et al: Protective effect of proeconditioning with PEP-1-CAT fusion protein against myocardial ischemiareperfusion injury in rats. Nan Fang Yi Ke Da Xue Xue Bao 2009, 29:2429-2432.

9. Pchejetski D, Kunduzova O, Dayon A, Calise D, Sequelas $M H$, et al: Oxidative stress-dependent sphingosine kinase-1 inhibition mediates monoamine oxidase A-associated cardiac cell apoptosis. Circ Res 2007, 100:41-49.

10. Huang GQ, Wang JN, Tang JM, Zhang L, Zheng F, et al: The combined transduction of copper, zinc-superoxide dismutase and catalase mediated by cell-penetrating peptide, PEP-1, to protect myocardium form ischemia-reperfusion injury. J Trans/ Med 2011, 9:73.

11. Yao LL, Wang JN, Huang YZ, Guo LY: Construction of prokaryotic expression plasmid pET15b-PEP-1-CAT and expression and purification of PEP-1-CAT fusion. Nan Fang Yi Ke Da Xue Xue Bao 2006, 26:1319-1325.

12. Shin EJ, Schram K, Zheng XL, Sweeney G: Leptin attenuates hypoxia/ reoxygenation-induced activation of the intrinsic pathway of apoptosis in rat H9c2 cells. J Cell Physiol 2009, 221:490-497. 
13. Lu N, Liu J, Liu J, Zhang C, Jiang F, et al: Antagonist effect of triptolide on AKT activation by truncated retinoid X receptor-alpha. PLoS One 2012, 7:e35722.

14. Kwon SH, Hong SI, Kim JA, Jung YH, Kim SY, et al: The neuroprotective effects of Lonicera japonica THUNB. against hydrogen peroxide-induced apoptosis via phosphorylation of MAPKs and PI3K/Akt in SH-SY5Y cells. Food Chem Toxicol 2011, 49:1011-1019.

15. Shirito K, Otani H, Yamamoto F, Huang CK, Maulik N, et al: MK2-/- gene knockout mouse hearts carry anti-apoptotic signal and are resistant to ischemia reperfusion injury. J Mol Cell Cardiol 2005, 38:93-97.

16. Yang W, Arai A, Aoki M, Ichijo H, Miura O: ASK1 is activated by arsenic trioxide in leukemic cells through accumulation of reactive oxygen species and may play a negative role in induction of apoptosis. Biochem Biophys Res Commun 2007, 355:1038-1044.

17. Bienengraeber M, Ozcan C, Terzic A: Stable transfection of UCP1 confers resistance to hypoxia/reoxygenation in a heart-derived cell line. $J \mathrm{Mol}$ Cell Cardiol 2003, 7:861-865.

18. Woo YH, Waye MM, Tsui SK, Yeung ST, Cheng CH: Andrographolide upregulates cellular-reduced glutathione level and protects cardiomyocytes against hypoxia/reoxygenation injury. J Pharmacol Exp Ther 2008, 325:226-235.

19. Becker LB: New concepts in reactive oxygen species and cardiovascular reperfusion physiology. Cardiovasc Res 2004, 61:461-470

20. Hirota Y, Kang D, Kanki T: The physiological role of mitophagy: new insights into phosphorylation events. Int I Cell Biol 2012, 2012:354914.

21. Park $M$, Youn $B$, Zheng $X L$, Wu D, Xu D: Globular adiponectin, acting via AdipoR1/APPL1, protects H9c2 cells from hypoxia/reoxygenationinduced apoptosis. PLoS One 2011, 6:e19143.

22. Cillick K, Crompton M: Evaluating cytochrome $\mathrm{c}$ diffusion in the intermembrane spaces of mitochondria during cytochrome $\mathrm{c}$ release. J Cell Sci 2008, 121:618-626.

23. Yurkova N, Shaw J, Blackie K, Weidman D, Jayas $R$, et al: The cell cycle factor E2F-1 activates Bnip3 and the intrinsic death pathway in ventricular myocytes. Circ Res 2008, 102:472-479.

24. Singh BK, Tripathi M, Chaudhari BP, Pandey PK, Kakkar P: Natural terpenes prevent mitochondrial dysfunction, oxidative stress and release of apoptotic proteins during nimesulide-hepatotoxicity in rats. PLoS One 2012, 7:e34200.

doi:10.1186/1479-5876-11-113

Cite this article as: Zhang et al:: PEP-1-CAT protects hypoxia/ reoxygenation-induced cardiomyocyte apoptosis through multiple sigaling pathways. Journal of Translational Medicine 2013 11:113.

\section{Submit your next manuscript to BioMed Central and take full advantage of:}

- Convenient online submission

- Thorough peer review

- No space constraints or color figure charges

- Immediate publication on acceptance

- Inclusion in PubMed, CAS, Scopus and Google Scholar

- Research which is freely available for redistribution 